\title{
Wavelength Demultiplexing Structure Based on Coupled-Cavity Waveguides in Photonic Crystals
}

\author{
A. MARTINEZ \\ J. MARTÍ
}

Fibre-Radio Group, ITACA Research Institute

Universidad Politecnica de Valencia, Valencia, Spain

\section{J. BRAVO-ABAD \\ J. SÁNCHEZ-DEHESA}

Dpto. Física Teórica de la Materia Condensada, Facultad de Ciencias (C-5) Universidad Autonoma de Madrid, Madrid, Spain

\begin{abstract}
A structure based on photonic crystals for optical wavelength demultiplexing is proposed and analyzed. This structure consists of two coupled-cavity waveguides, each one tuned at a different frequency transmission band. A model based on the tightbinding method taken from solid-state physics is used to design the working frequencies of the waveguides. The demultiplexing behavior is demonstrated by means of simulations employing a finite-difference time-domain method. It is concluded that the demultiplexing mechanism presented here may be used as a building block in multifunctional lightwave integrated circuits based on photonic crystals.
\end{abstract}

Keywords photonic crystals, wavelength demultiplexers, coupled-cavity waveguides

\section{Introduction}

Photonic crystals (PhCs) are envisaged as the main candidates for developing microscale integrated lightwave circuits because of their properties for controlling the flow of light on a very small scale $[1,2]$. The periodic change in the refractive index of these artificial materials gives rise to photonic band gaps (PBGs) where no electromagnetic modes can propagate inside the crystal. By inserting linear defects into PhCs, highly confined propagating states that can propagate lossless even through sharp bends appear inside the PBG [3]. In this manner, two-dimensional (2D) PhC optical waveguides based on the PBG effect have been proposed. In such 2D waveguides, light is confined in the direction normal to the $2 \mathrm{D}$ PhC plane by means of total internal reflection [4]. The performance of straight and sharply bent waveguides at optical frequencies has been evaluated by means of simulations [5] and experimental results [6]. Recently, a different kind of PhC-

Received 23 October 2002; accepted 21 December 2002.

Address correspondence to Alejandro Martinez, ITACA Research Institute, Universidad Politecnica de Valencia, Camino de Vera s/n, 46022 Valencia, Spain. E-mail: almarab@itaca.upv.es 
based waveguide commonly known as a coupled-resonator optical waveguide (CROW) [7] or coupled-cavity waveguide (CCW) [8] has been proposed in which light guiding is explained as photon hopping between nearly confined states around point defects.

On the other hand, several PhC-based optical demultiplexing approaches based on waveguide to high-Q cavity coupling [9-10], superprism phenomena [11], and waveguide couplers [12] have been reported. The main advantages of such approaches are the extremely small size of the structures and the possibility of integrating the structure in multifunctional PhC-based devices. In this work we propose a $1 \times 2$ demultiplexing structure that relies on the use of two CCWs tuned at different frequencies by modifying the cavities of the waveguide. Some advantages of this structure are straightforward design rules, ultra-small size, very low cross-talk, and enough physical separation of the output channels. This last property may allow an easy interconnection to optical fibers or planar dielectric waveguides. This structure may be used, for instance, as a first wideband demultiplexing stage in multifunctional PhC-based lightwave integrated circuits.

\section{Single-Line Defect and Coupled-Cavity Waveguides}

First, let us analyze the transmission properties of $\mathrm{PhC}$ waveguides in a hexagonal 2D PhC consisting of silicon rods $(\varepsilon=12)$ in air $(\varepsilon=1)$ in which the relation between the radius of the rods $(r)$ and the lattice constant $(a)$ is $r / a=0.15$. The plane-wave expansion (PWE) method [13] is used to obtain the photonic bands of the ideal periodic structure and the waveguides. The frequencies of the modes are calculated in normalized frequency units $f=a / \lambda, \lambda$ being the wavelength in vacuum. PWE results show that a wide PBG appear for TM modes. The midgap frequency is $f_{g}=0.4239$ and the relative PBG width is $\Delta f / f_{g} \approx 0.47$.

If an entire row of rods is removed, a single-line defect waveguide (SLDW) is obtained, as is shown in Figure 1(a), where a waveguide is observed along the $\Gamma \mathrm{K}$ direction of the hexagonal lattice. However, if only one of every two rods of the entire row is removed, a CCW is formed, as shown in Figure 1(b). Light propagation in CCWs is achieved by photon hopping between nearest-neighbor cavities because of the overlapping of the modes localized in the defects.

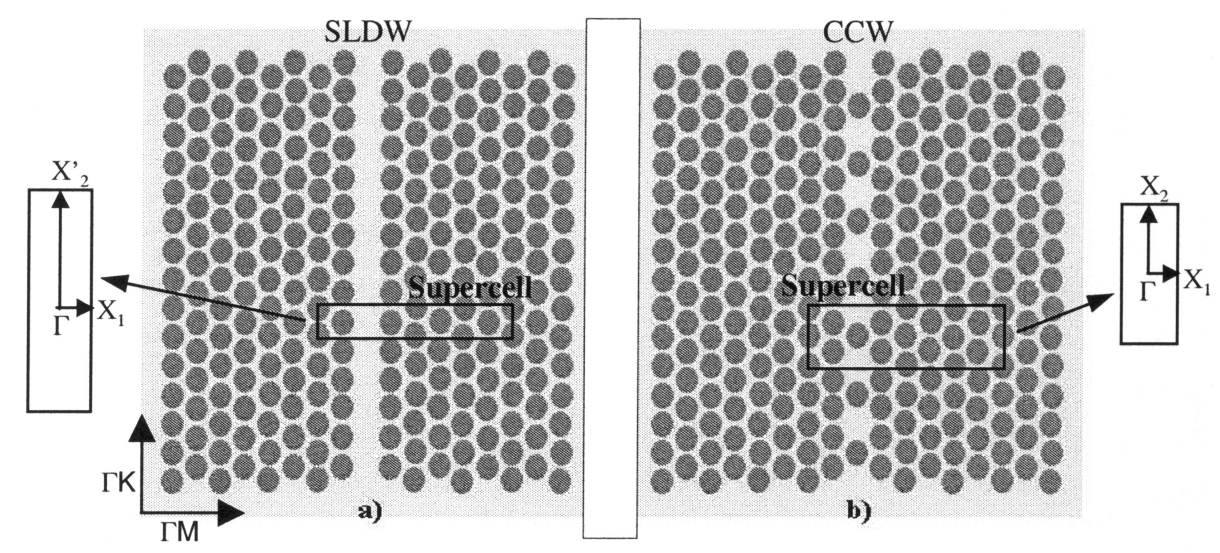

Figure 1. Scheme of a single-line defect waveguide originated from removing an entire row of dielectric rods (a) and a coupled-cavity waveguide in which only one of each two rods of the row is removed (b). The supercell used in the band structure calculations, its Brillouin zone, and the high-symmetry points of the rectangular reciprocal lattices are also shown. 
Figures 1(a) and 1(b) also show the supercells used to calculate the dispersion relations of the waveguides as well as their corresponding Brillouin zones. The $\Gamma \mathrm{K}$ direction in the hexagonal structure turns into the $\Gamma \mathrm{X}_{2}^{\prime}$ and $\Gamma \mathrm{X}_{2}$ directions in the rectangular Brillouin zones for the SLDW and the CCW, respectively. The high-symmetry points along the waveguide direction are $\mathrm{X}_{2}=\pi / 2 a$ and $\mathrm{X}_{2}^{\prime}=\pi / a$ in the reciprocal space. Taking into account only the direction $\Gamma \mathrm{K}$, the TM PBG ranges from 0.3209 to 0.5235 in $(a / \lambda)$ units. The resulting dispersion relations of both waveguides are shown in Figure 2. Notice that the bandwidth of the $\mathrm{CCW}\left(\Delta f_{\mathrm{CCW}}=0.0225\right)$ (see Figure 2(a)) is narrower than the one of the SLDW $\left(\Delta f_{\text {SLDW }}=0.1159\right)$ (see Figure 2(b)). This can be explained by considering that a SLDW also consists of a chain of cavities resulting from removing a rod but in this case without any rod separating two consecutive cavities. Then the coupling between cavities is stronger than in the CCW case, so the SLDW bandwidth is larger. A tight-binding (TB) model [7] taken from solid-state physics can be employed to fit the dispersion relation of the guided modes of a chain of defect cavities. The guided bands can be cast into the following expression:

$$
f(k)=f_{0}\left(1+\kappa_{1} \cos (k d)\right),
$$

where $f$ is the frequency of the guided mode, $f_{0}$ is the frequency of the fundamental level of an isolated cavity, $\kappa_{1}$ is the hopping parameter that quantifies the strength of the intercavity coupling, $k$ is the wavevector, and $d$ is the distance between consecutive defects ( $d=2 a$ in the PhC described above). Let us remark that $f_{0}$ is obtained from PWE calculations by using a large supercell (35 cylinders basis) in which a single defect is embedded. Therefore, $\kappa_{1}$ remains as the only fitting parameter in the model, which is related to the bandwidth of the guided modes $\Delta f$ by means of the following expression (see Equation (1)):

$$
\left|\kappa_{1}\right|=\frac{\Delta f}{2 f_{0}} .
$$
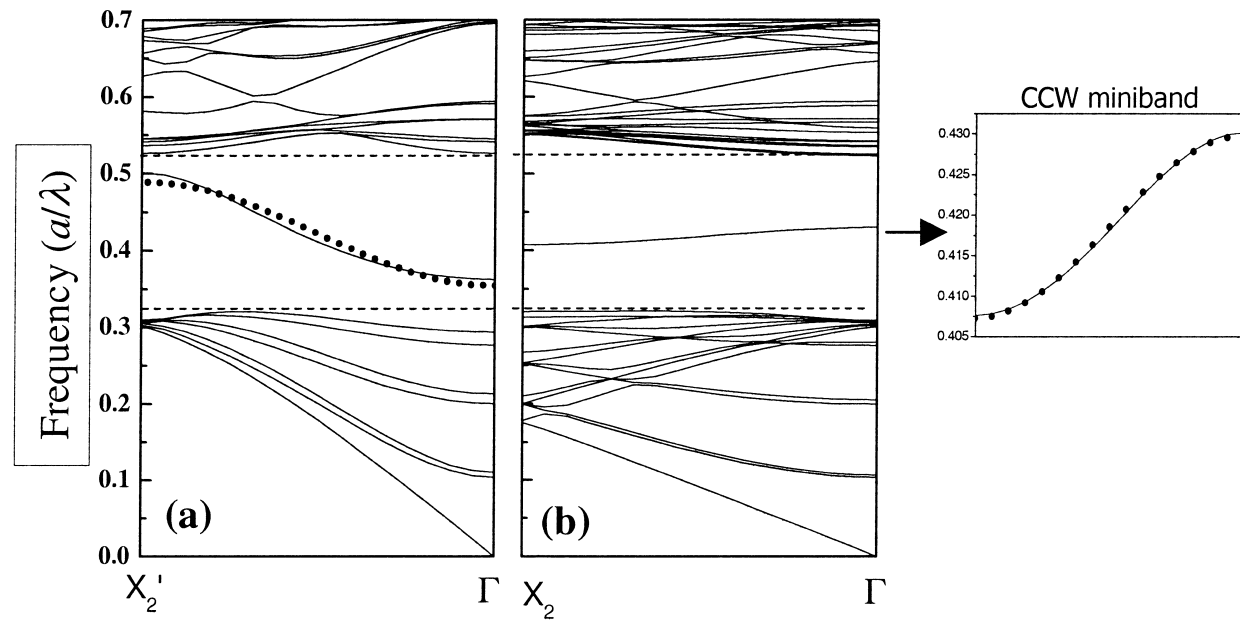

Figure 2. Dispersion relation of a single-line defect waveguide (a) and a CCW with $r_{\text {def }}=0$ (b) along the direction $\Gamma X_{2}$. Solid lines stand for PWE calculations, and dotted lines represent the bands fitted with the TB model. The inset shows an enlarged view of the CCW miniband. 
The dotted lines in Figure 2 represent the fitted TB bands. The central frequency, corresponding to the eigenfrequency of an isolated cavity, is $f_{0} \approx 0.4185$ for both waveguides while the hopping parameter is $\left|\kappa_{1}\right| \approx 0.1385$ for the SLDW and $\left|\kappa_{1}\right| \approx$ 0.0268 for the CCW, respectively. It is remarkable that the good fitting was obtained in both cases with our single parameter. The fitting to the miniband in SLDW could be enhanced by including more parameters in the fitting procedure, in other words, to use Equation (1) with additional terms.

\section{Tunability of Coupled-Cavity Waveguides}

Equation (1) indicates that the transmission behavior of a CCW depends mainly on two parameters: the frequency of the isolated defect, which determines the central frequency of the CCW miniband, and the hopping parameter, which determines its width. So if the cavity is modified, its eigenfrequency changes and the CCW miniband moves along the frequency axis in the photonic band structure. Moreover, if the distance between cavities is increased the coupling becomes weaker, and the width of the CCW miniband becomes smaller. Therefore, a CCW can be seen as an optical bandpass filter that can be tuned by modifying the structure of the cavities and the distance between them. From now on, it will be considered that the CCW defects are Si rods whose radius $r_{\text {def }}$ is lower than the radius of the rest of the rods. The distance between cavities is assumed to remain constant $(d=2 a)$ in this analysis. Figure 3 shows the variation of the central frequency of the CCW miniband $\left(f_{0}\right)$ as a function of the radius of the defect rod for several values of the $r / a$ parameter obtained from PWE simulations. From Figure 3 it can be noticed that when $r_{\text {def }}$ increases, the miniband shifts toward lower frequencies (higher wavelengths), as expected from the electromagnetic variational theorem [3].

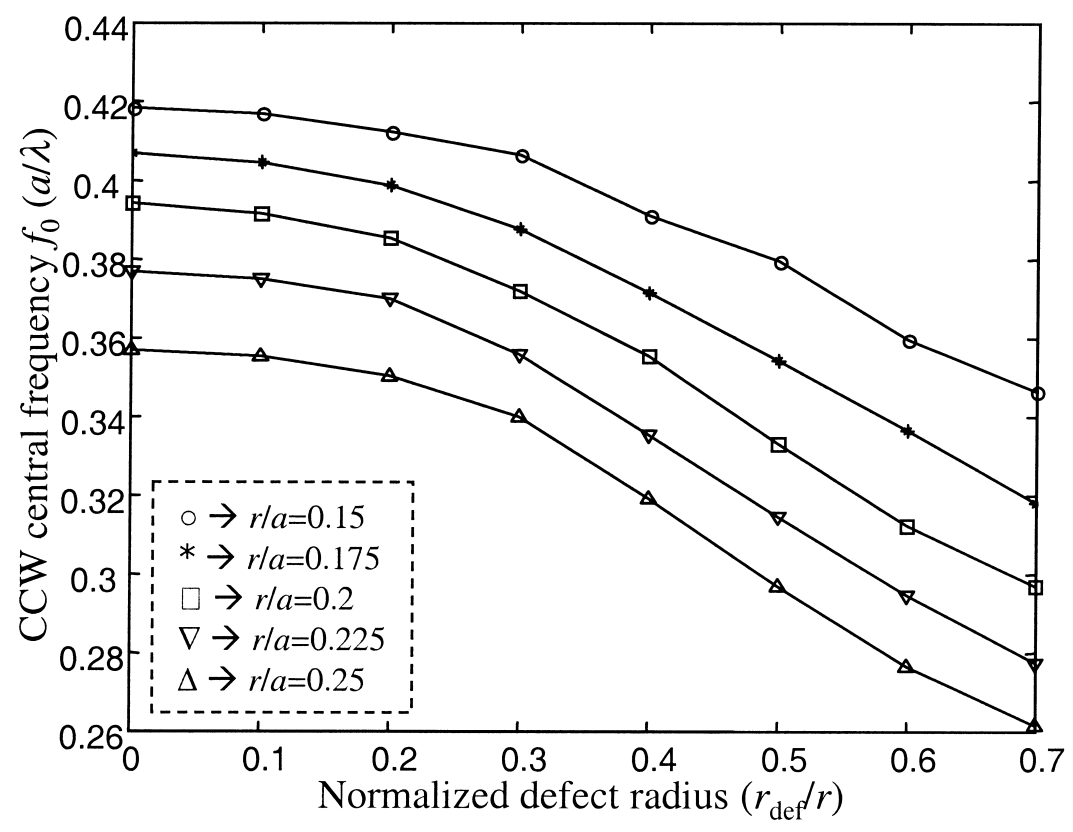

Figure 3. Normalized central frequency of the $\mathrm{CCW}$ miniband as a function of the radius of the defect for several $r / a$ ratios. 


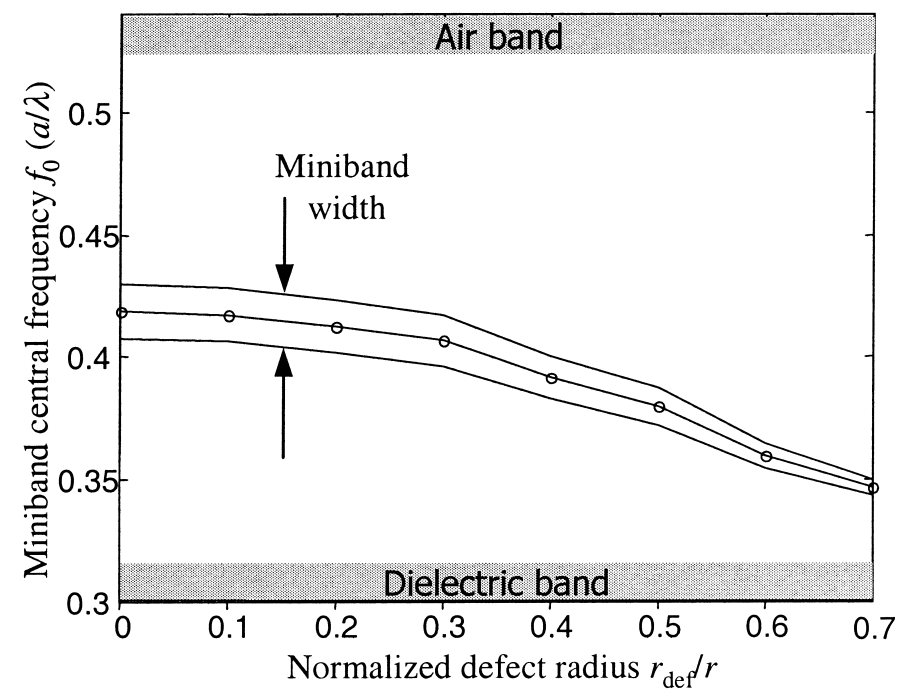

Figure 4. Variation of the central frequency and the width of the CCW miniband as a function of the radius of the defects for a fixed ratio $r / a=0.15$. The shaded regions define the air (bottom) and dielectric (top) bands.

However, not only does the central frequency of the CCW miniband diminish as $r_{\text {def }}$ increases, but the CCW bandwidth also decreases, because the modes are more confined in the cavities, so the intercavity coupling becomes weaker. This can be observed in Figure 4, which shows the variation of the central frequency and the bandwidth of the CCW miniband as a function of $r_{\text {def }}$ for the case $r / a=0.15$.

\section{Transmission Characterization of Coupled-Cavity Waveguides}

The transmission response of a SLDW and a CCW has been obtained by using the finite-difference time-domain (FDTD) method [14]. To this purpose, a 2D finite cell of the considered $\mathrm{PhC}$ with waveguides along the $\Gamma \mathrm{K}$ direction of the hexagonal lattice has been employed. Perfectly matched layer conditions are applied at the cell boundaries [15]. In what follows, frequencies are denormalized in order to treat a case of practical application and make the results easier to understand. A lattice constant $a=550 \mathrm{~nm}$ is used, which means that for a CCW with $r_{\mathrm{def}}=0$, a passband centered at $1,310 \mathrm{~nm}$ is achieved, while the TM PBG corresponding to the hexagonal lattice is defined between 1,050 and $1,717 \mathrm{~nm}$. A Gaussian wide-band optical pulse is used as input signal to study the transmission of light through the waveguides. Figure 5 shows the normalized transmission spectra of a SLDW (solid line) and a CCW with $r_{\mathrm{def}}=0$ (dashed line). It is noticed that the bandwidth of the SLDW is larger than the CCW bandwidth because of the closer distance between cavities, confirming the predictions of the PWE method and the TB model. The central frequencies of the guided modes of both waveguides agree well with the PWE results. Losses in both waveguides are mainly due to the index mismatching between the input and output ends and the waveguides. The ripples in both transmission spectra are due to the finiteness of the waveguides, that is, the finite number of cavities. Thus, as the simulated CCW consists of eight cavities, its transmission spectrum shows eight frequency peaks (see the inset in Figure 5). Nevertheless, taking into account the 


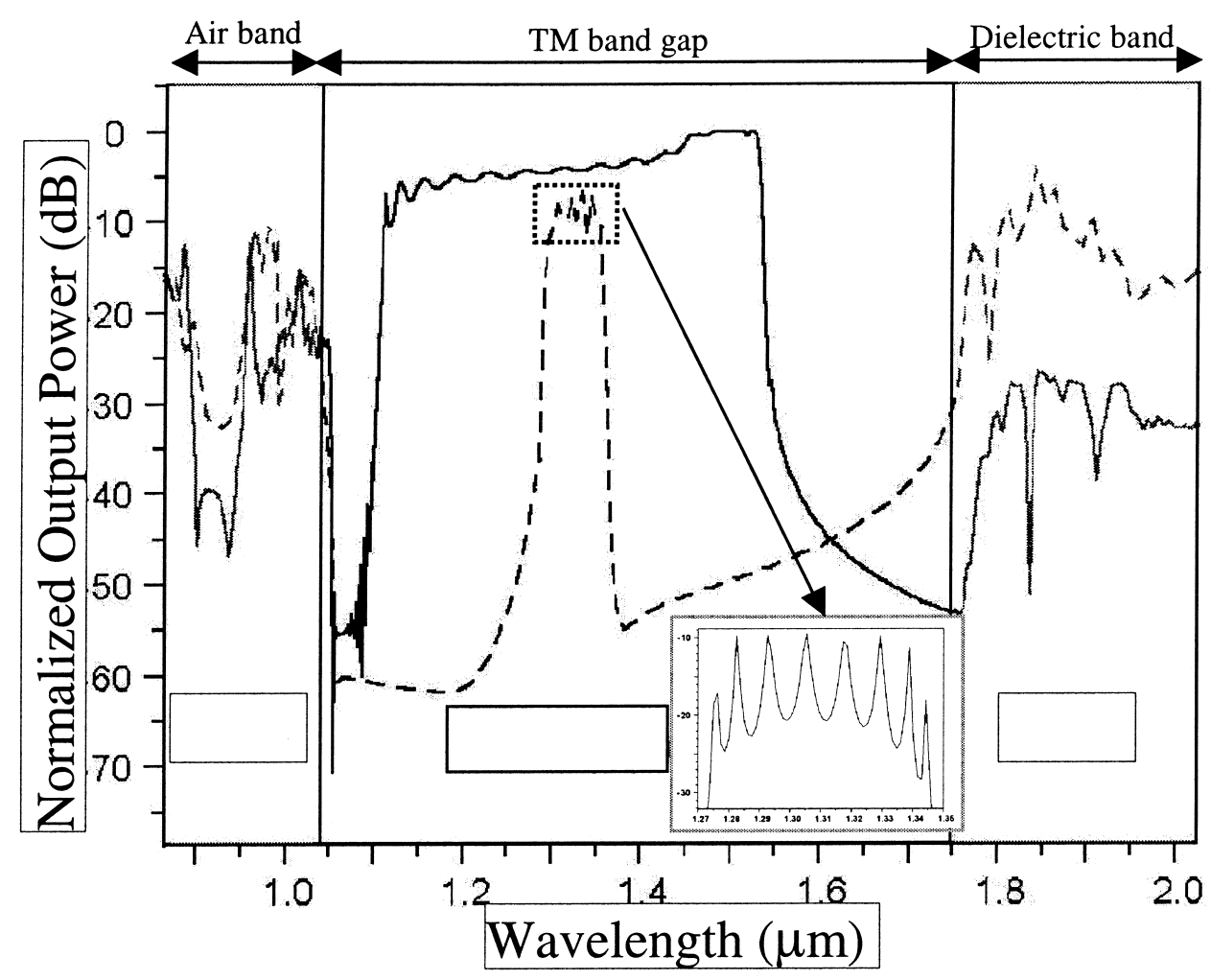

Figure 5. Normalized transmission spectra of an SLDW (solid line) and a CCW with $r_{\text {def }}=0$ (dashed line). The inset shows a detailed profile of the CCW spectrum in which eight frequency peaks appear clearly.

theoretical model proposed in [16], it can be assumed that by increasing the number of cavities, the degradation caused by the ripple can be overcome.

\section{Demultiplexing Structure}

Figure 6 shows a scheme of the demultiplexing structure that we propose from the results reported above. First, light travels along a SLDW that afterwards is split in two different CCWs (CCW 1 and CCW 2). Because of its large bandwidth (see Figure 5), the SLDW is designed to support all the frequencies that can propagate through both CCWs. The input and both output waveguides are allocated along the $\Gamma \mathrm{K}$ direction of the hexagonal lattice. Output CCWs must be designed in a way that allows the transmission of different nonoverlapping frequency bands in order to obtain a right demultiplexing performance.

As an example, the two CCWs have been designed to split the second and third optical transmission windows, although other frequency ranges may have been chosen. The lattice parameter and the radius of the rods are kept as in the previous calculations ( $a=550 \mathrm{~nm}, r / a=0.15$ ). Under this consideration, CCW 1 and CCW 2 are designed to transmit the $1,310 \mathrm{~nm}$ and the $1,550 \mathrm{~nm}$ bands, respectively, so the radius of the defects are taken $r_{1}=0$ (no column inside the cavity) for CCW 1 and $r_{2}=46 \mathrm{~nm}$ for CCW 2 by taking into account PWE predictions shown in Figure 3. 


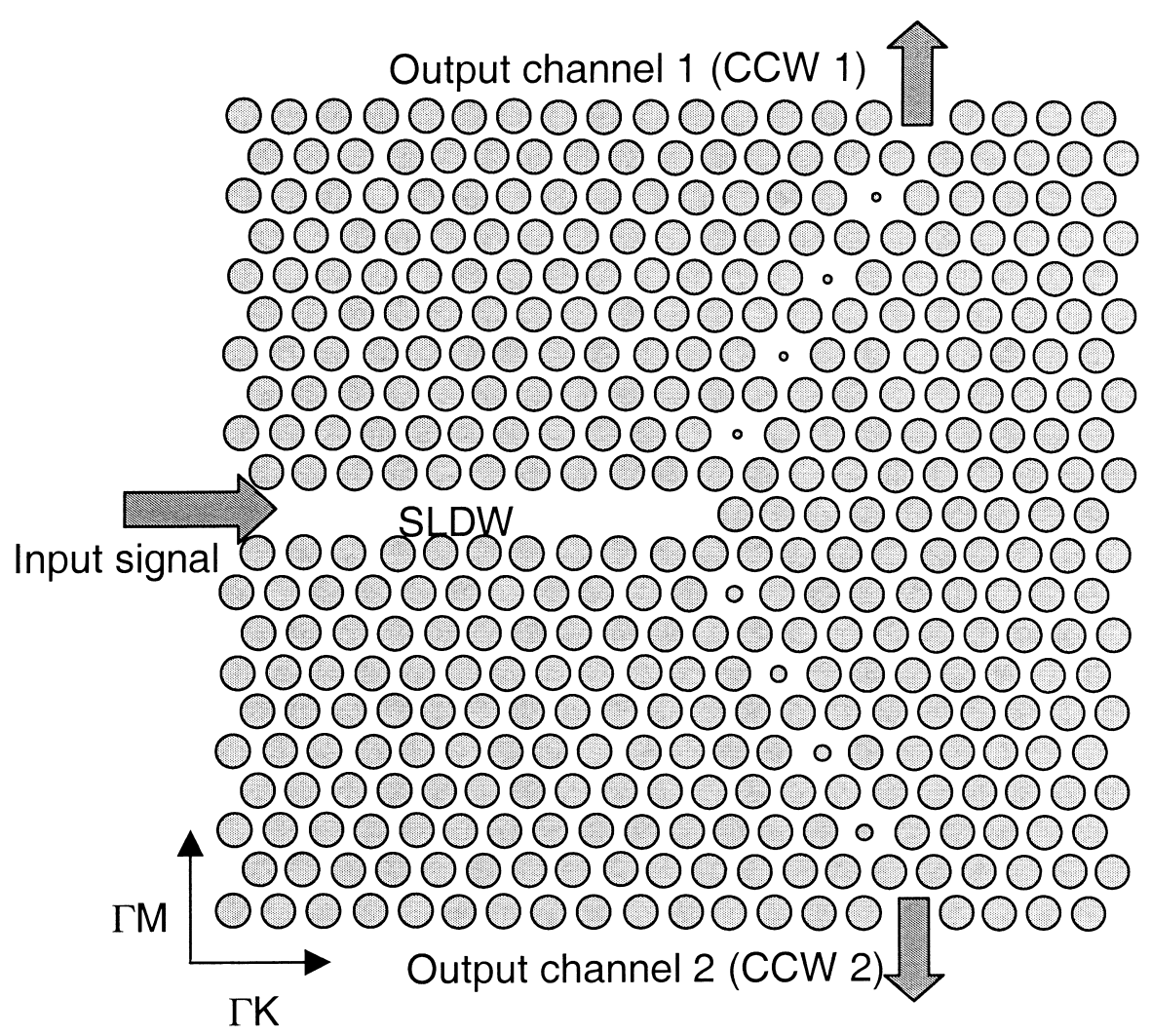

Figure 6. Scheme of the proposed PhC-based $1 \times 2$ demultiplexing structure. An input SLDW is split in two CCWs designed to transmit different frequency bands.

FDTD simulations were carried out by injecting a Gaussian optical pulse into the SLDW, and the optical power is monitored at both outputs. Figure 7 shows the normalized transmission spectra at the output of both the upper (dotted line) and the lower (solid line) branches. The bandwidth of both upper and lower branches are around 69 and $43 \mathrm{~nm}$, respectively, so, in principle, this mechanism is suitable for wideband demultiplexing. Cross-talk, defined as the ratio between the power of the undesired and desired bands at the outputs, is around $-60 \mathrm{~dB}$ for the $1,310 \mathrm{~nm}$ branch and $-55 \mathrm{~dB}$ for the $1,550 \mathrm{~nm}$ branch and may be improved if the ripple due to a finite quantity of cavities is reduced by lengthening the waveguides. The main loss sources are the mismatching at both ends, because light is coupled from and to vacuum, and the wave vector mismatching between the SLDW and each CCW, which may be minimized by properly designing the splitting region [17]. Propagation losses may be considered negligible. Because of the rotational symmetry of the cavity modes, the propagation through the bends of each CCW should be lossless over its entire bandwidth, since the coupling of a corner cavity to its two consecutive cavities is identical [7].

Finally, Figure 8 shows the propagation of the electric field component parallel to the column axis for two monochromatic waves with different wavelengths ((a) $1,320 \mathrm{~nm}$ and (b) $1,545 \mathrm{~nm}$ ) injected in the structure. The demultiplexing effect is clearly observed. 


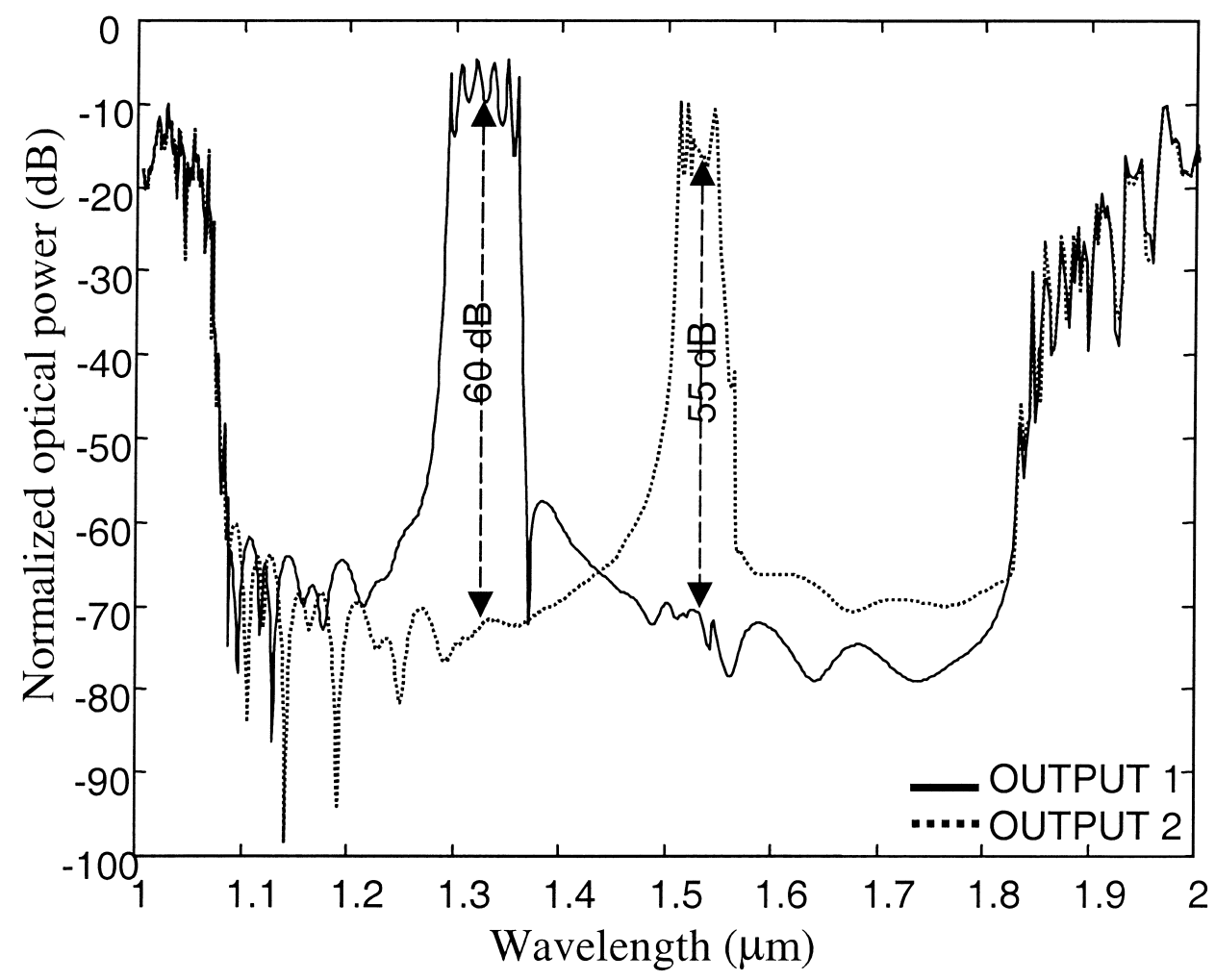

Figure 7. Normalized transmission spectra of the left branch (solid line) and the right branch (dashed line) of the demultiplexing structure. Cross-talk in both branches is indicated.
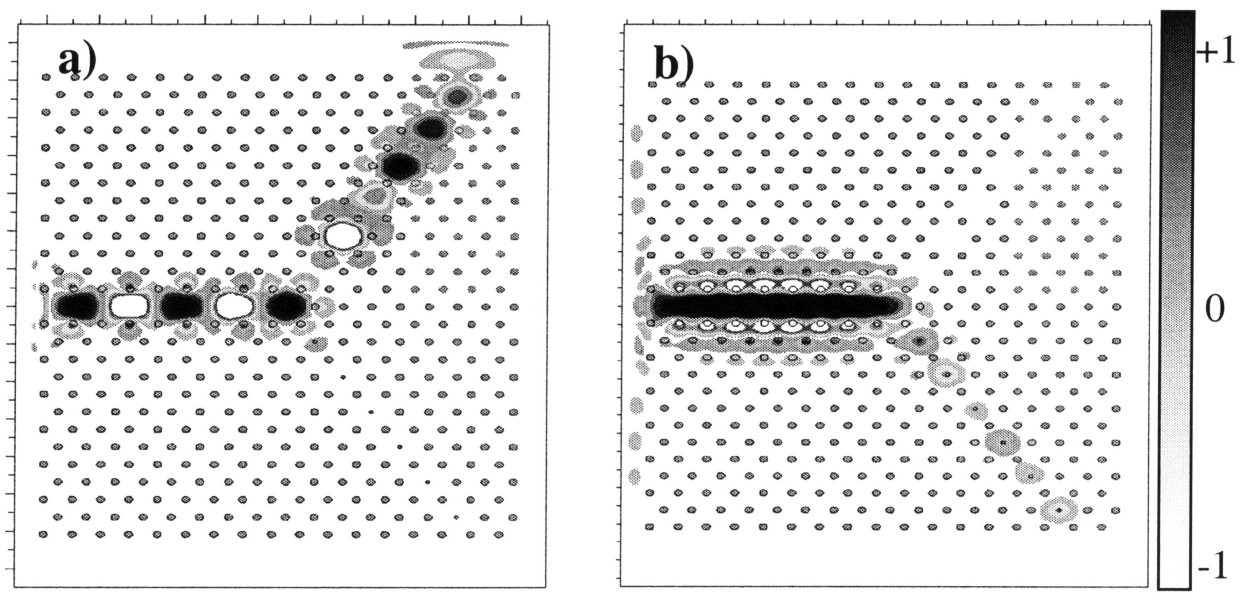

Figure 8. Real part of the electric field component parallel to the dielectric rods for two different monochromatic waves: (a) $\lambda=1,320 \mathrm{~nm}$ and (b) $\lambda=1,545 \mathrm{~nm}$. The demultiplexing behavior of the structure is demonstrated. 
Let us stress that the same demultiplexing principle described above may be applied to a $2 \mathrm{D} \mathrm{PhC}$ consisting of cylindrical holes on a dielectric substrate if the waveguides are made single-mode, which can be achieved by employing several methods, such as that explained in Notomi et al. [18].

\section{Summary}

A wavelength demultiplexing structure based on 2D photonic crystal waveguides has been introduced and its performance analyzed. This structure is mainly suitable for wideband signals. Nevertheless, the flexibility inherent to coupled-cavity waveguides makes the design also useful for signals with a narrower bandwidth by enlarging the distance between cavities. $1 \times N$ demultiplexers may also be obtained by cascading several $1 \times 2$ structures. The main drawbacks of this structure are the losses expected from the mismatching between the input and both output waveguides and the ripple of the transmitted band induced by the finiteness of the CCWs. Both drawbacks may be overcome: the first by employing matching techniques, and the second by lengthening the waveguides.

\section{References}

1. Yablonovitch, E. 1987. Inhibited spontaneous emission in solid-state physics and electronics. Phys. Rev. Lett. 58:2059-2062.

2. John, S. 1987. Strong localization of photons in certain disordered dielectric superlattices. Phys. Rev. Lett. 58:2486-2489.

3. Joannopoulos, J. D., R. D. Meade, and J. N. Winn. 1995. Photonic Crystals: Molding the Flow of Light. Princeton: Princeton University Press.

4. Krauss, T. F., R. M. De la Rue, and S. Brand. 1996. Two-dimensional photonic-bandgap structures operating at near infrared wavelengths. Nature 383:699-702.

5. Mekis, A., J. C. Chen, I. Kurland, S. Fan, P. R. Villeneuve, and J. D. Joannopoulos. 1996. High transmission through sharp bends in photonic crystal waveguides. Phys. Rev. Lett. 77:37873790.

6. Baba, T., N. Fukaya, and J. Yonekura. 1999. Observation of light propagation in photonic crystal optical waveguides with bends. Electron. Lett. 35:654-655.

7. Yariv, A., Y. Xu, R. K. Lee, and A. Scherer. 1999. Coupled-resonator optical waveguide: A proposal and analysis. Opt. Lett. 24(11):711-713.

8. Bayindir, M., B. Temelkuran, and E. Ozbay. 2000. Tight-binding description of the coupled defect modes in three-dimensional photonic crystals. Phys. Rev. Lett. 84:2140-2143.

9. Noda, S., A. Chutinan, and M. Imada. 2000. Trapping and emission of photons by a single defect in a photonic bandgap structure. Nature 407:608-610.

10. Fan, S., P. R. Villeneuve, J. D. Joannopoulos, and H. A. Haus. 1998. Channel drop filters in photonic crystals. Optics Express 3(1):4-11.

11. Kosaka, H., T. Kawashima, A. Tomita, M. Notomi, T. Tamamura, T. Sato, and S. Kawakami. 1999. Superprism phenomena in photonic crystals: Toward microscale lightwave circuits. J. Lightwave Technol. 17:2032-2038.

12. Koshiba, M. 2001. Wavelength division multiplexing and demultiplexing with photonic crystal waveguide couplers. J. Lightwave Technol. 19:1970-1975.

13. Johnson, S. G., and J. D. Joannopoulos. 2001. Block-iterative frequency-domain methods for Maxwell's equations in a planewave basis. Opt. Express 8:173-190.

14. Yee, K. S. 1966. Numerical solution of initial boundary value problems involving Maxwell's equations in isotropic media. IEEE Trans. Antennas Propagat. AP-14:302-307.

15. Berenger, J. P. 1994. A perfectly matched layer for the absorption of electro-magnetic waves. J. Comput. Phys. 114:185. 
16. Reynolds, A. L., U. Peschel, F. Lederer, P. J. Roberts, T. F. Krauss, and P. J. I. de Maagt. 2001. Coupled defects in photonic crystals. IEEE Trans. Microwave Theory Tech. 49(10):1860.

17. Boscolo, S., M. Midrio, and T. F. Krauss. 2002. Y-junctions in photonic crystal channel waveguides: High transmission and impedance matching. Opt. Lett. 27(12):1001-1003.

18. Notomi, M., A. Shinya, K. Yamada, J. Takahashi, C. Takahashi, and I. Yokohama. 2001. Singlemode transmission within photonic bandgap of with-varied single-line-defect photonic crystal waveguides on SOI substrates. Electron. Lett. 37(5):293-295.

\section{Biographies}

Alejandro Martinez received the Ingeniero de Telecomunicación degree in 2000 from the Universidad Politecnica de Valencia. He is currently working towards his $\mathrm{PhD}$ degree and is a member of the Fiber-Radio Group. His research interests include applications of microwave and millimeter-wave radio over fiber systems as well as planar photonic crystals and their applications in optical networks.

Javier Martí received the Ingeniero de Telecomunicación degree from the Universidad Politécnica de Catalunya, Catalunya, Spain, in 1991, and the Doctor Ingeniero de Telecomunicación degree ( $\mathrm{PhD}$ ) from the Universidad Politécnica de Valencia, Valencia, Spain, in 1994. During 1989 and 1990, he was an Assistant Lecturer at the Escuela Universitaria de Vilanova, Barcelona, Spain. From 1991 to 2000, he was a Lecturer and Associate Professor at the Telecommunication Engineering Faculty, where he is currently a Professor and leads the Fiber Radio Group. He has authored or coauthored over 100 articles in refereed international technical journals and over 50 articles in international conference proceedings in the fields of broadband hybrid fiber-radio systems and microwave/millimeter-wave photonics, fiber-based access networks, terabit/s OTDM/WDM optical networks, advanced optical processing techniques for microwave signals, and ultrahigh-speed data transmission and planar photonic crystals. Prof. Martí has served as member of the Technical Program Committee of ECOC, Microwave Photonics and other international workshops and conferences.

Jorge Bravo-Abad was born in Madrid, Spain, in 1978. He received the BS degree in condensed matter physics from the Autonomous University of Madrid in 2001. He is currently working towards his $\mathrm{PhD}$ degree at the Autonomous University of Madrid.

Jose Sánchez-Dehesa was born in Manzaneque, Toledo, Spain, in 1955. He received the Doctoral degree in Physics from the Autonomous University of Madrid, Spain, in 1982. Since 1985 he has been associated professor at the Autonomous University of Madrid. He has worked on structures based on semiconductors, such as quantum wells, superlattices, quantum wires, etc. Now his work is mainly related with the propagation of electromagnetic waves in photonic crystals, as well as in the propagation of elastic waves in phononic crystals. 